\title{
Review of: "Effect of antioxidant treatment with n- acetylcysteine and swimming on lipid expression of sebaceous glands in diabetic mice"
}

\author{
Emanuela Camera, Giorgia Cardinali ${ }^{1}$ \\ 1 Hospitaller Physiotherapeutic Institutes (IFO)
}

Potential competing interests: The author(s) declared that no potential competing interests exist.

Diabetes is a disease consequent to the impaired response of tissues to insulin. The irresponsiveness of the insulin receptor results into deranged carbohydrate metabolism, hyperglycemia and tissue damage. Diabetogenic diets have been shown to contribute to acne, which is the major disorder of the sebaceous gland (SG) in humans $\left({ }^{[1]}\right)$. The SG is a holocrine gland in the skin that manufactures and secretes a lipidrich fluid known as sebum, which serves several functions in preserving skin integrity. Increasing body of evidence is placing SG in the wider context of extra-cutaneous metabolic processes. The paper addressed the interesting subject of the SG modifications and sebaceous lipid accumulation in diabetic conditions. However, the data presented appear to be rather preliminary in terms of experimental approaches and performed evaluations.

The study was comprised of two objectives. The first one was to investigate effects of the glutathioneinducing n-acetylcysteine (NAC) together with swimming exercise on the SG morphology, and lipogenic activity. The second one was methodological in nature as it was meant to assess the advantage of histochemistry $(\mathrm{HC})$ or immunohistochemistry $(\mathrm{IHC})$ reactions towards SG's lipids, performed with sudan black and adipophilin (ADPH) staining, respectively. The overlap of methodological aspects and experimental observations was tortuous. Comparison of HC and IHC in the evaluation of SG morphology and lipid content indicated that each one had its own advantage. IHC of ADPH showed a good straining of intracytoplasmic lipids and membranes of intracytoplasmic vesicles. HC with sudan black showed strong marking, which possibly offered a more sensitive approach to detect lipids in small quantity. The enlarged boxes displayed on the pictures were difficult to connect with the corresponding tissue shown at lower magnification. Nevertheless, the comparison between sudan black and ADPH staining offered a technical strategy that, in our opinion, cannot be considered an objective of the study. The experimental design itself involved 25 female mice (15-week-old and weight average $20 \mathrm{~g}$ ). Of these, 20 were non-obese diabetic (NOD) mice and 5 inbred BALB/c mice. Thus, the mice undergoing specific intervention were all non-obese and diabetic. Each group consisted of 5 mice. The NOD group was divided in: UD, untreated group; DS, mice practicing swimming exercise; DNa, consisting of NOD mice treated with NAC; DS-Na, mice practicing swimming and assuming NAC. The hyperglycemic and diabetic conditions were assumed based on the urine excretion of glucose. DS mice had urine levels higher than $300 \mathrm{mg} / \mathrm{dL}$. A clear 
understanding of the experimental design required the integration of information provided in the abstract with that reported in the methods section. The Authors demonstrated the positive effect of swimming activity in tissue recovery, but no mechanistic investigations were attempted to explain the ineffectiveness of the NAC treatment in the DNa group and the aggravating effects in the DS-Na group.

Two important points were missing in the data interpretation. Effects of NAC are multiple and difficult to capture in their entirety ${ }^{[2]}$ ). However, providing readouts of NAC supplementation was not attempted. NAC was selected for its capacity to increase GSH synthesis. Evidence that the expected effects of NAC had occurred or not were fundamental to interpret the results. Useful information could derive from the positive effects observed with swimming exercise alone. Insulin sensitivity has important implications in the processes governing sebocyte differentiation and balanced lipid synthesis in the SG ${ }^{\left[{ }^{[3]}\right)}$. Perturbation of the normal differentiation program of the SG associates with derangement in the lipid composition ( ${ }^{[4]}$ ). Data on sebum lipid composition would be a useful complement to the observations on SG morphology. Information on improved sensitivity to insulin after exercise was also important to support interpretation of the results. Lastly, exercise is a fat burning practice. Whether or not body weight and SG density were changed in the different groups could assist interpretation.

\section{References}

1. Ailary Baldwin, Jerry Tan. (2020). Effects of Diet on Acne and Its Response to Treatment. Am J Clin Dermatol, vol. 22 (1), 55-65. doi:10.1007/s40257-020-00542-y.

2. ^Lesley I. McLellan, Alexander D. Lewis, David J. Hall, John D. Ansell, et al. (1995). Uptake and distribution of $\mathrm{N}$-acetylcysteine in mice: tissue-specific effects on glutathione concentrations. Carcinogenesis, vol. 16 (9), 2099-2106. doi:10.1093/carcin/16.9.2099.

3. ^A. Mastrofrancesco, M. Ottaviani, G. Cardinali, E. Flori, et al. (2017). Pharmacological PPARY modulation regulates sebogenesis and inflammation in SZ95 human sebocytes. Biochemical Pharmacology, vol. 138 , 96-106. doi:10.1016/j.bcp.2017.04.030.

4. `Karmveer Singh, Emanuela Camera, Linda Krug, Abhijit Basu, et al. (2018). JunB defines functional and structural integrity of the epidermo-pilosebaceous unit in the skin. Nat Commun, vol. 9 (1). doi:10.1038/s41467-018-05726-z. 